Journal of Computer Science 8 (6): 913-919, 2012

ISSN 1549-3636

(C) 2012 Science Publications

\title{
The Comparison of Experimental and Analytical Study of the Gaussian IntensityDistribution for Light Emitting Diodes Beam
}

\author{
Mohammad Syuhaimi Ab-Rahman, \\ Luqman Al-Hakim Azizan, Harry Ramza and Zulkifli Musa \\ Department of Electrical, Electronics and System Engineering, \\ Faculty of Engineering and Built Environmental, \\ Space Science Institute (ANGKASA), University Kebangsaan, Malaysia
}

\begin{abstract}
Problem statement: Wireless communication using white Light Emitting Diodes (LEDs) is the latest research field for next-generation communication. This study studies the comparison of Gaussian intensity distribution of the white LED using experimental and analytical method. The white LEDs are conducted to transmit an audio signal to the receiver. The receiver circuit consist of solar cell connected to the speaker to recover the audio signal. From the comparison of experimental and analytical data, the Gaussian plot of experimental data is steeper than the analytical data, meaning that the LED has small-divergence beam. Conclusion/Recommendations: The output voltage of experimental works decrease exponentially with the distance whiles the Full Width Half Maximum (FWHM) value increase exponentially with the distance. The gradual increment and decrement of the analytical signal can be applicable to visible light communication implementation as such light source can cover wide area for signal transmission.
\end{abstract}

Key words: Wireless communication, white LED, solar cell, visible light, Gaussian intensity distribution, Full Width Half Maximum (FWHM)

\section{INTRODUCTION}

Lighting industries the world over are moving forward with the production of white Light Emitting Diodes (LEDs) for illumination, replacing fluorescent and incandescent bulbs. The white LED is expected to be the next-generation lighting source because of its numerous advantages over other technologies. Compared with conventional lighting devices, white LEDs exhibit low power consumption, long life expectancy, high reliability, small size and high tolerance to humidity. Compared with normal lights, another important property of LEDs is their ability to be modulated at high speeds. This ability makes it possible for LEDs to be used as signal transmitters. It is useful for short-range communication systems that could be implemented in indoor and outdoor applications (Tanaka et al., 2000; Komine and Nakagawa, 2004; Tanaka et al., 2003), which are currently dominated by Radio Frequency (RF) communication.

The ability of LEDs to be signal transmitters means that LEDs could act as dual-function devices for both lighting and communication. Wireless communication that relies on light is known as "Visible Light Communication". The visible light spectrum offers unlimited bandwidth and is unregulated worldwide. Using visible light for indoor communication is secure from eavesdropping, because visible light cannot penetrate walls or other opaque barriers. Signal transmission is confined to the room in which the signals originate and interference between links operating in different rooms is thus prevented.

The use of RF for communications is restricted in specific areas, such as hospitals and on flights, because of the effect of interference on precision equipment. This study presents a basic experiment that demonstrates audio transmission using a white LED. Currently, short-range audio transmission can be accomplished using the RF technology known as Bluetooth. This study discusses basic experimental work that was carried out to demonstrate the use of LED beams to realise wireless communication. The success of this experiment is an indicator that futuregeneration communication systems will no longer rely on RF but will instead utilise visible light.

\section{Overview:}

Infrared Vs visible light communication: As explained by (Khan and Barry, 1997), both visible and infrared light offer unlimited bandwidth and high-speed 
transmission. Some characteristics of LEDs and Laser Diodes (LDs) are quite similar, but there are still differences between the electromagnetic waves the two types of diodes produce. The major difference is that the optical output of the LED is incoherent, while that of the LD is coherent (Keiser, 2008). Moreover, infrared radiation can present human eye safety hazards, because it can pass through the human cornea and be focussed by the lens onto the retina, where it can potentially induce thermal damage (Rancourt, 1993). This means that visible light has more potential for use in indoor short-range communication because it is not harmful to humans.

The progress of visible light communication: Keiser (2008) began his study by noting the rapid spread of research and development of visible light communication (Nakagawa, 2007; Langer et al., 2008; 2009; Conti, 2008), as well as the growth of the worldwide LED illumination market. In this study, he proposed a new photodetection technique using image processing instead of PIN-photo diodes or avalanche photo diodes (Keiser, 2008). According to (Keiser, 2008), using a high-speed sensor with a frame rate of thousands of frames per second enables data rates on the order of kilobits per second (Keiser, 2008). An indoor optical wireless duplex communication system was introduced by (Rui et al., 2009), who proposes a system using white LEDs as the emitters in the downlink and infrared LEDs in the uplink. Through experiments in a prototype room, they discovered that the LED emission properties, the positions of multiple light sources and path differences in diffuse channels were the three main factors influencing the pulse response.

Gaussian LED beams: The Full Width Half Maximum (FWHM) describes the measured width of the Gaussian graph, as shown in Fig. 1. The w stands for the beam waist and the FWHM is equal to $\mathrm{w}$ times 2. Figure 2 shows the characteristic of an LED beam that produces incoherent optical output. The incoherent source has a broad spectral width because the optical energy is emitted into a hemisphere according to a cosine power distribution (Keiser, 2008). Therefore, the LED beam will propagate with a large divergence. The large divergence of the beam makes LEDs appropriate for illuminating wide areas for human activity. The Gaussian profile indicates that the strength of the beamlike wave falls off in the transverse direction according to a bell-shaped curve that is symmetrical around the central axis, illustrated in Fig. 1 (Hecht, 2008). A Gaussian function of a variable is the negative exponential of the square of that variable, which in this case is the distance ( $r$ ) in a transverse plane from the central axis of propagation (z) (Hecht, 2008).

\section{Experimental work:}

Transmitter and receiver circuit: In wireless communication systems, there are only two components that influence the output efficiency of the signal: the transmitter and the receiver. In an optical communication system, the output signal produced at the receiver must be the same as the input signal being transmitted.

Figure 3 is a block diagram of the entire optical audio transmission system. The audio signal is encoded by the encoder and injected to the current amplifier and the LED driver. The LED driver is then connected to the LED and the signal is transmitted using the LED's light spectrum. The light propagates to the photodetector, which acts as the receiver. At the receiver, the signal passes through a filter to filter the noise. Next, the signal is amplified and decoded back to an analogue signal. Finally, the amplified signal is connected to the speaker. A comparator is used to calculate the speed of the link between the transmitter and the receiver.

The circuit shown in Fig. 4 was constructed and tested in the laboratory and some of its characteristics were analyzed. The basic circuit used a super-bright LED as the modulator. This transmitter works by modulating the amplitude of the light based on the amplitude of the audio signal being transmitted. The receiver was built using a solar cell and a speaker was connected to it.

A standard $3.5 \mathrm{~mm}$ audio jack feeds the audio signal to the circuit, which is sent to the 470-ohm resistor, a switch and a 9-volt battery. The battery provides a steady DC current to the LED and causes it to glow with a fixed brightness. The resistor's function is to limit the current so the LED does not burn out, whereas the switch is used to control the circuit.

A weak, fluctuating radio signal is added to the constant signal from the battery after the audio jack is connected to the audio source. The LED still glows, but now it blinks synchronously with the audio signal as the current passing through it varies. Electrical signals are generated when the blinking light hits the solar cell. This signal again varies in synchronisation with the original audio signal. The signals are then fed to the speaker, recreating the original sounds from the audio source.

The experimental setups are shown in Fig. 5-8 show photographs of the actual setups taken during the experiments.

The value of the output voltage according to the position of light on the receiver is recorded for analysis. By varying the vertical position $X$ and the horizontal position $\mathrm{Y}$, the effectiveness of the photocell and the relationship between the position and the induced voltage can be studied. The receiver is connected to the amplified speaker and a multimeter. Observations are made based on the data collected and the output audio signal heard. 


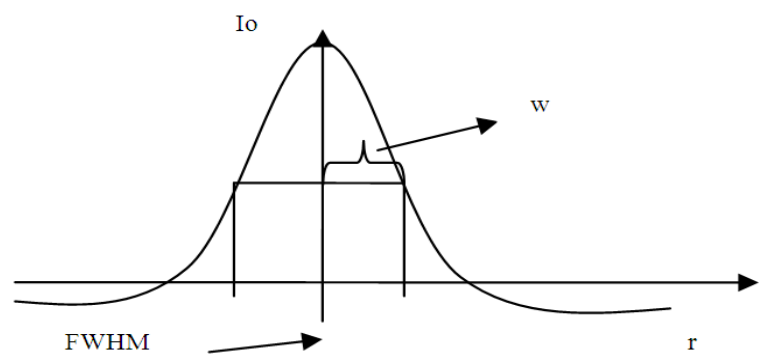

Fig. 1: Gaussian beam-like wave propagation

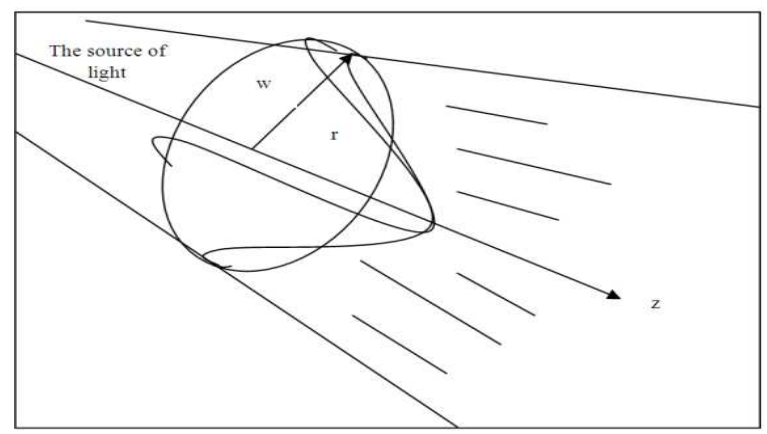

Fig. 2: The light beam produced by an LED propagating through air

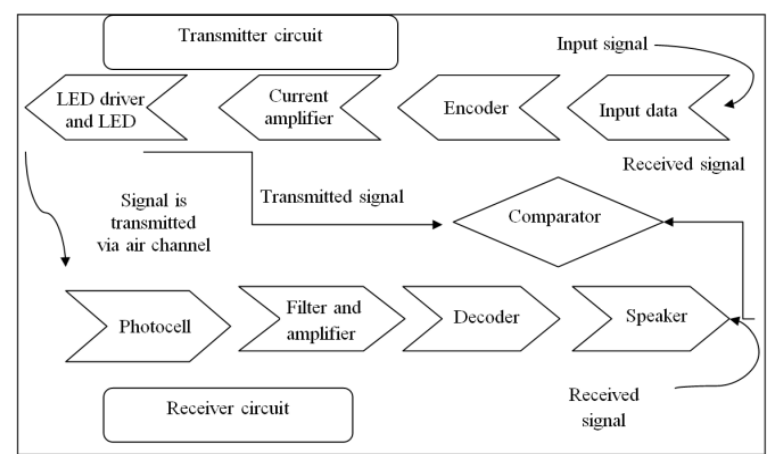

Fig. 3: A diagram of the transmitter and receiver of a visible light communication system

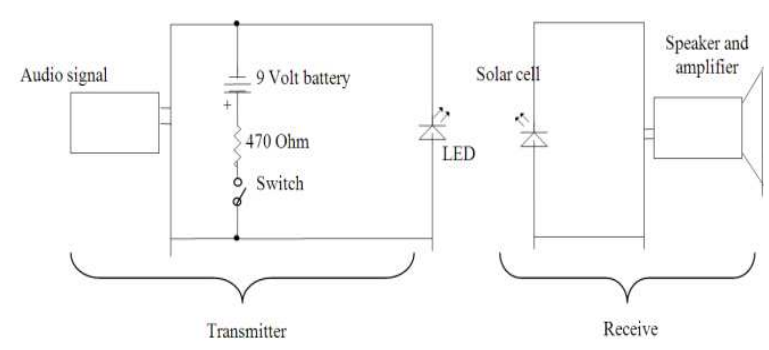

Fig. 4: Circuit used to carry out the experiments in the laboratory.

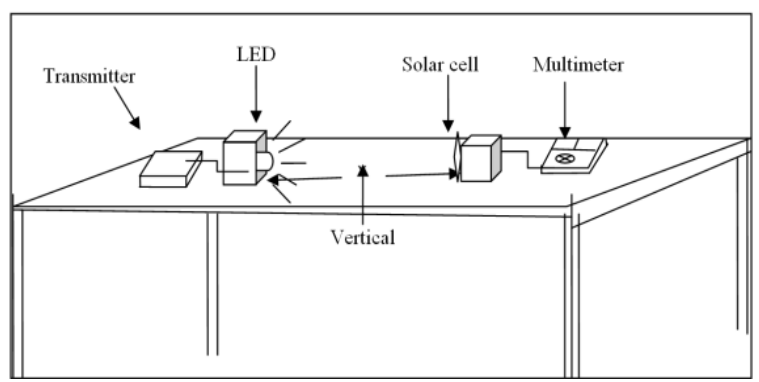

Fig. 5: Experimental setup to measure the relationship between the vertical distance $\mathrm{X}$ and the output voltage

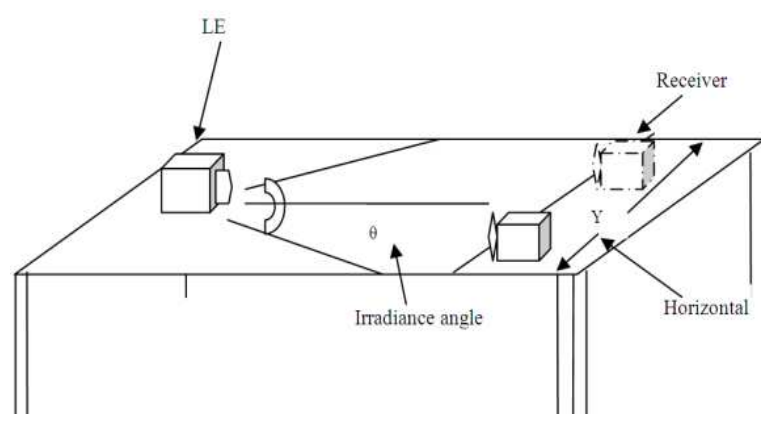

Fig. 6: Experimental setup to measure the relationship between the horizontal distance, $\mathrm{Y}$ and the received voltage

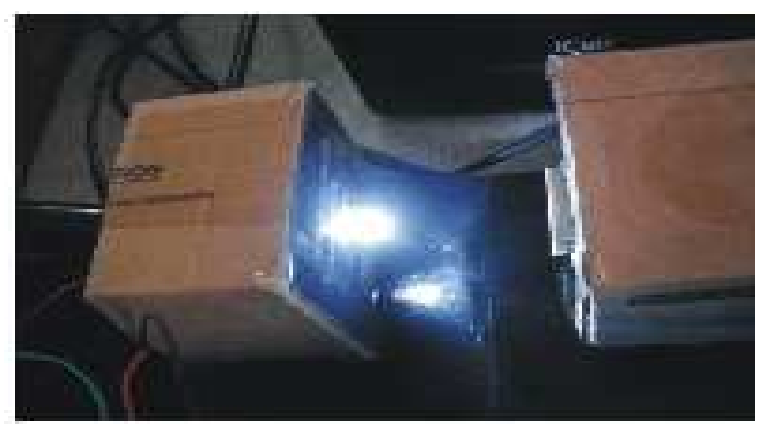

Fig.7: The LED shines directly on the photocell

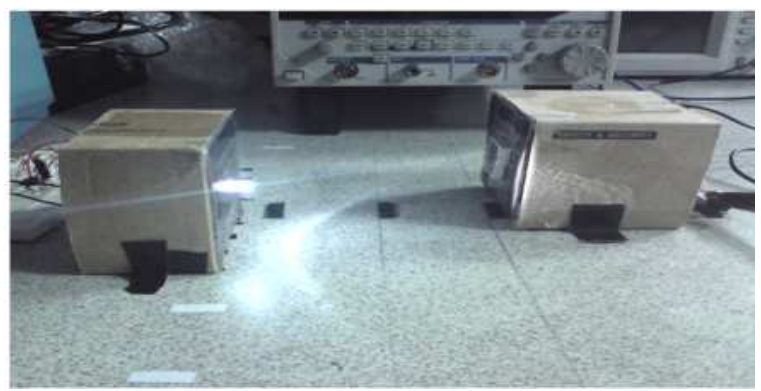

Fig. 8: A photo taken of the experimental setup of Fig. 6 
The results: The basic circuit in Fig. 4 was tested by measuring the output voltage while varying 2 parameters: the horizontal distance $\mathrm{Y}$ and the vertical distance X. The setups are shown in Fig. 5 and 6. Eq. 1, shown below, was used to calculate the analytical value of the output voltage in the form of an electric field (Es). The experimental and analytical results were compared and analyzed (Hecht, 2008):

$$
\mathrm{Es}=\mathrm{Eo} \times \mathrm{e}\left(\mathrm{r}^{2} / \mathrm{w}^{2}\right)
$$

$\mathrm{R}$ refers to the distance between the transmitter and the receiver, while $w$ represents the width of the LED spectrum. Let $r=w$ be the beam's half-width, the distance at which the electric field of the beam drops from its maximum axial value Eo to $37 \%$ of Eo (Hecht, 2008). In the graph below, the Y position is negative so that the Gaussian distribution is evident in the graph of the output voltage. The centre of the receiver is at the value of $Y=0$ and the plots extend $6 \mathrm{~cm}$ to the left and right.

From Eq. 1, the intensity can be calculated using a second Eq. 2 (Hecht, 2008):

$$
\text { Is }=\eta \times \operatorname{Eo} \times \operatorname{Eoe}^{-}\left(\frac{2^{-2}}{w^{2}}\right)
$$

The electric field is measured in units of voltage and compared with the experimental result, as explained in the observation and discussion sections. The second observation is the output audio signal, which could be heard as the distance varied.

Graphs of observations: The graphs in Fig. 9, labelled a-g, present a number of observations. These observations demonstrate that the vertical distance $\mathrm{X}$ (Fig. 5) and the horizontal distance Y (Fig. 6) affect the output voltage value. Data were measured in 2 experimental setups, one in which the $\mathrm{X}$ position was varied and the $\mathrm{Y}$ position was fixed and another in which the $\mathrm{Y}$ position was varied and the $\mathrm{X}$ position was fixed, as shown in Fig. 5 and 6. Instead of observing the output voltage as a function of these distances, the output audio signal could also be observed. The Gaussian intensity distribution graphs in Fig. 9a-g show the observed and analytical distributions of light as the horizontal distance $\mathrm{Y}$ was varied at a fixed vertical distance, as shown in Fig. 6.

The results show that the highest value of the output voltage, $0.175 \mathrm{~V}$, was obtained at a vertical distance $\mathrm{X}$ of $5 \mathrm{~cm}$ and a horizontal distance $\mathrm{Y}$ of 0 (at the centre), as shown in Fig. 9a. At this peak point, the output audio sound was clear. The comparison in Fig. 9a reveals a slight difference in the peak experimental and analytical values. The experimental result also deviated significantly from the analytical result as the output voltage was very sensitive to changes in position. In other words, the observations revealed much steeper increments and decrements than the analytical result. The output sound could be recovered from the speaker up to $\mathrm{Y}$ distances of $6 \mathrm{~cm}$ to the left and right, but the quality of the signal was low.

Figure $9 \mathrm{~b}$ shows a comparison of the experimental and analytical outputs at a vertical distance $X$ of $10 \mathrm{~cm}$. The difference of the peak output voltages of the two graphs is $10 \mathrm{mV}$. The graph of the experimental data increases and decreases more drastically than the analytical graph. The highest output voltage was obtained at a horizontal distance $\mathrm{Y}$ of $0 \mathrm{~cm}$ (at the centre). At this point, the output sound could be heard clearly. The experimental setup seems to be very sensitive to the changes in the horizontal position. At a vertical distance of $X=10 \mathrm{~cm}$, the sound could be heard out to $\mathrm{Y}$ positions $6 \mathrm{~cm}$ to the left and right, but the sound was unclear and of low quality.

In Fig. 9c, the distance between the transmitter and receiver was increased to $X=15 \mathrm{~cm}$. The received output voltage and the quality of the output audio signal were lower than at $X$ values of 5 and $10 \mathrm{~cm}$, shown in Fig. 9a and $b$, respectively. The difference between the analytical and experimental peak output voltages is $31 \mathrm{mV}$. The experimental graph increases and decreases significantly as the horizontal distance $Y$ varies $6 \mathrm{~cm}$ to the left and to the right and the intensity of light received at the photodetector at the maximum $Y$ distances $(-6$ and $6 \mathrm{~cm})$ is very low. The output audio sound could only be heard out to $\mathrm{Y}$ positions $4 \mathrm{~cm}$ from the centre.

In Fig. 9d, the vertical distance $X$ is fixed at $20 \mathrm{~cm}$. The difference in the analytical and experimental peak voltages is $37 \mathrm{mV}$, which is larger than those shown in Fig. 9a-c. As before, the graph of the analytical data increases and decreases gradually, whereas the experimental graph increases and decreases sharply. The value of the experimental data at the maximum horizontal distances -6 and $6 \mathrm{~cm}$ is zero, while the analytical value is $29 \mathrm{mV}$ at the same Y positions. A voltage of zero means that the receiver did not receive any light and the receiver produced no output audio. The audio signal could still be heard at $\mathrm{Y}=0$ $\mathrm{cm}$, but the quality was lower than it was in the setups of Fig. 9a-c.

Figure 9e shows the analytical and experimental results at a distance of $X=25 \mathrm{~cm}$. The analytical graph increases and decreases gently as the horizontal position varies, but the experimental graph is again steeper and the difference in the peak output voltages is $35 \mathrm{mV}$. The experimental peak value is $60 \mathrm{mV}$ at the position $\mathrm{Y}=0$. The output audio signal produced by the receiver could be heard until the $\mathrm{Y}$ position reached $4 \mathrm{~cm}$ from the centre. 


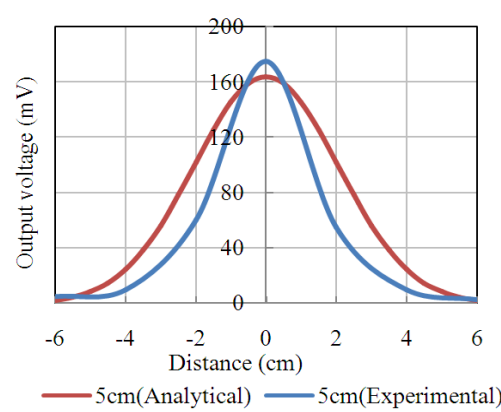

(a)

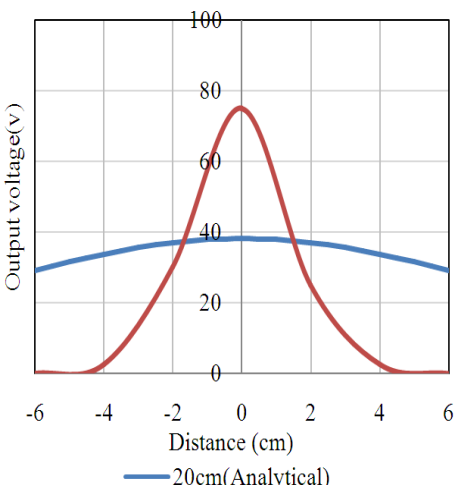

(d)

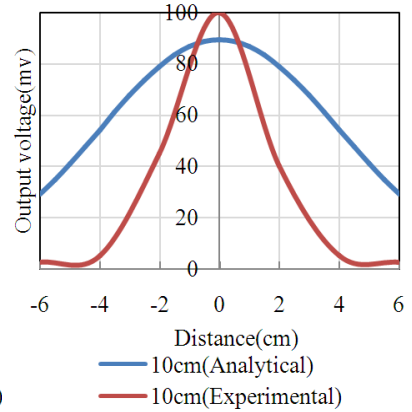

(b)

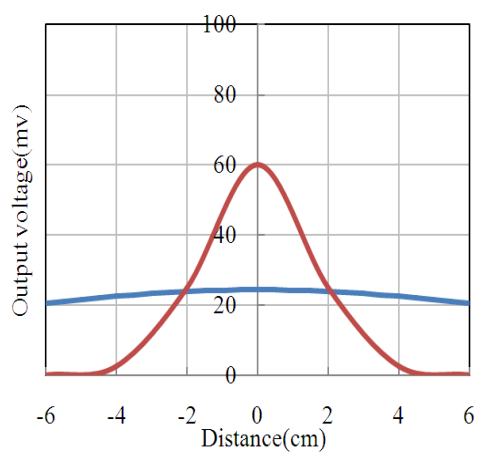

$-25 \mathrm{~cm}$ (Analytical) $-25 \mathrm{~cm}$ (Experimental)

(e)

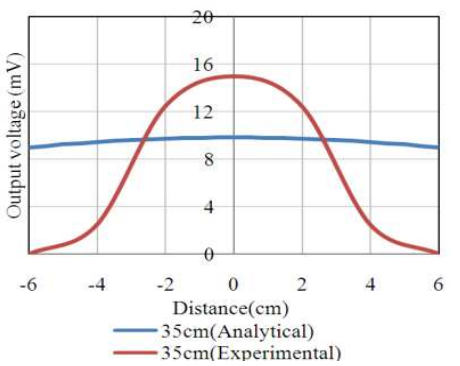

(g)

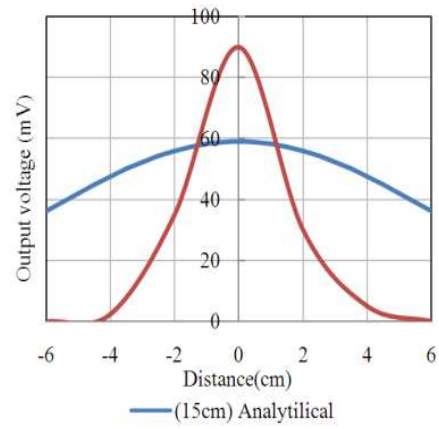

(c)

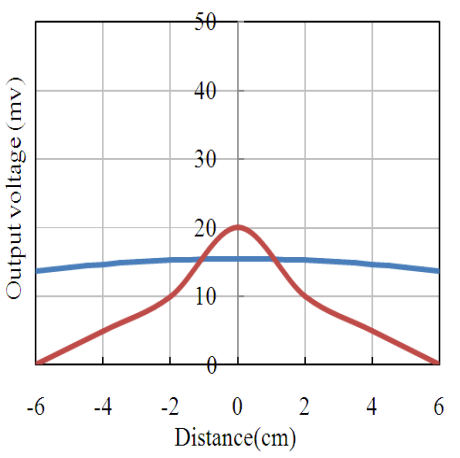

$30 \mathrm{~cm}$ (Analytical)

(f)

Fig. 9: Comparison between the experimental and analytical graphs. At a distance of (a) $X=5 \mathrm{~cm}$, (b) $X=10 \mathrm{~cm}$, (c) $X=15 \mathrm{~cm}$, (d) $X=20 \mathrm{~cm}$, (e) $X=25 \mathrm{~cm}$, (f) $X=30 \mathrm{~cm}$ and (g) $X=35 \mathrm{~cm}$

In Figure 9f, the difference between the analytical and experimental peak output voltages was small. At the distance $X=30 \mathrm{~cm}$, the experimental graph increases gradually until Y positions approximately 2 $\mathrm{cm}$ from the centre and the graph becomes steeper near the peak. The decrement also follows the same trend: steep for the first $2 \mathrm{~cm}$ and more gradual as the distance increases. The difference between the peak voltages is only $5 \mathrm{mV}$. The sound quality produced by the speaker was low.

In Fig. 9g, at an X distance of $35 \mathrm{~cm}$, the increment and decrement of the experimental graph again becomes more gradual. The intensity of light received by the photocell was quite weak at this point and this can be seen in the resulting output voltage. The quality of the output audio signal was the lowest at this $\mathrm{X}$ distance. The audio signal could only be heard until Y positions $2 \mathrm{~cm}$ to the left and right of the centre. The analytical data again exhibits a gradual increment and decrement, as shown in Fig. 9b-f. Figure 10 shows the output voltage at $\mathrm{Y}=0$ as $\mathrm{X}$ varies from $5-35 \mathrm{~cm}$. The graph shows that the intensity decreases exponentially with the $\mathrm{X}$ distance. The data is plotted with the exponential model function of below Eq. 3:

$y=\alpha \times e^{b x}+c$ 


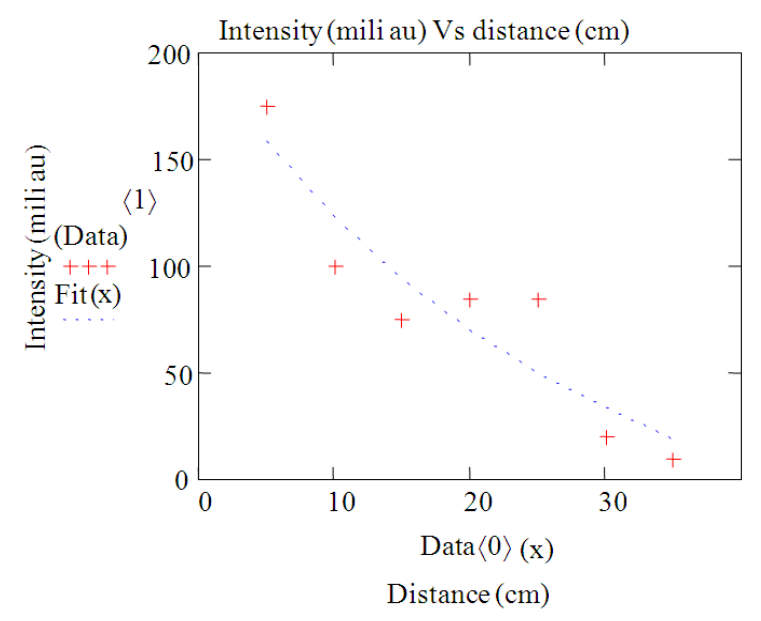

Fig. 10: The exponential shape of the output voltage at a fixed $\mathrm{Y}$ value $(\mathrm{Y}=0$, at the centre) as the vertical distance $X$ varies from $5-35 \mathrm{~cm}$

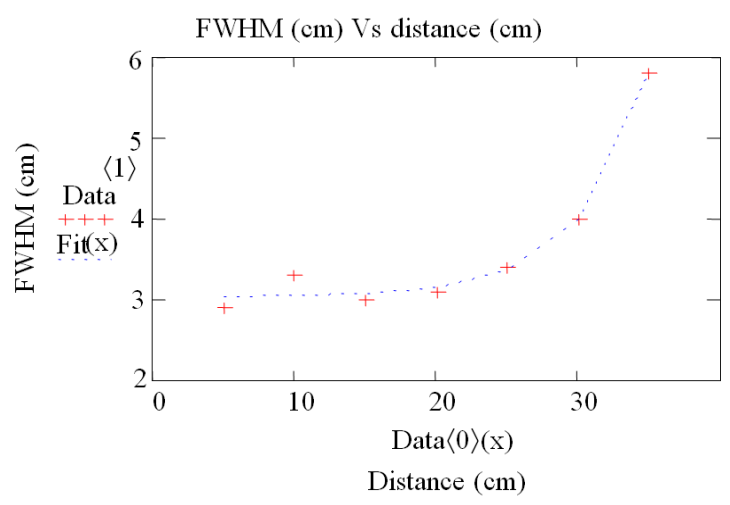

Fig. 11: An exponential graph of the FWHM versus the vertical distance $\mathrm{X}$

where, $a, b$ and $c$ is the coefficients of the graph. As the distance becomes large, the received output voltage becomes small. The received output voltage depends on the intensity of the light falling on the surface of the photodetector. The graph above come across of an Eq. 4 below:

$y=248.415 \times e^{-0.027 x}-47.25$

The equation relates that the initial value of intensity at the distance of 0 is approximately $\mathrm{y}=$ 248.415-47.25 that gives the value $2.011 \mathrm{mili}$ au. This graph is plotted in the MathCad software and, the value of coefficients a is 248.415 , b is -0.037 and $\mathrm{c}$ is -47.25 .

Figure 11 shows the full width at half maximum versus the vertical distance X. Generally, the full width at half maximum increased proportionally with the $\mathrm{X}$ distance. The data plotted in Fig. 11 shows that the relationship between FWHM and X is exponential and the model function is expressed. The equation that extracted from the Fig. 11 is as below Eq. 5:

$y=1.718 .10^{-3} \times e^{0.211 x}-3.037$

It means that at the distance of 0 , the value of FWHM is approximately $3.039 \mathrm{~cm}$. Applying the same model faction and same program of the graph in Fig. 10, the FWHM increased exponentially with the value of coefficients a, b and c are 0.00718, 0.211 and 3.037.

The graphs in Fig. 9b-g show that the experimental peak output voltage is higher than the analytical peak voltage because of the profile of the LED. Different LED profiles produce different results. Brighter LEDs will produce very high peak intensities on the photocell. The experimental voltage is a steeper function of the horizontal distance $\mathrm{Y}$ than the analytical voltage. The sensitivity to received light is related to the light beam produced by the LED and will decrease when the transmitted beam is wide. A wider light beam will allow the receiver to receive light at distances beyond 6 $\mathrm{cm}$ from the centre. The experimental results plotted in Fig. 9a-g exhibited Gaussian intensity distributions. Figure 10 revealed that the light intensity is reciprocal to the distance between the transmitter and receiver. The output audio signal could still be heard at a distance of $\mathrm{X}=35 \mathrm{~cm}$, but only at horizontal distances up to $2 \mathrm{~cm}$ from the centre as the received intensity decreased when the distance increased. The sound could still be heard until a distance of $50 \mathrm{~cm}$ if the transmitter and receiver were directly facing each other (Syuhaimi and Ab-Rahman, 2011).

The exponentially decrement of intensity of light versus the distance proving the theory of light that, as the source far apart from the receiver, the intensity become low. In Fig. 11, the full width at half maximum distribution explained the characteristics of the LED light used in this study. As the distance varied, the radius of the LED light became larger, because the LED beam diverges as it propagates (Keiser, 2008).

\section{CONCLUSION}

From the observations and the discussion of the graphs, we conclude that wide beams are needed in visible light communication systems to ensure that the light is able to brighten a wide area with high intensity. A wide, high-intensity beam will ensure that the transmitted signal can be received by a photodetector at any point in the illuminated area. The analytical graphs in Fig. 9 are more applicable than are the experimental 
graphs because they represent a beam with a large divergence. Moreover, large-divergence and highintensity beams will influence the values of the full width at half maximum shown in Fig. 11. The Full Width at Half Maximum (FWHM) represents the diameter of the LED beam. Figure 11 shows that the LED beam diverges strongly as it propagates because as the distance increased, the value of the FWHM also increased. A specific LED profile with high intensity and a broad beam should be used in visible light communication applications. In fact, the method of data collection in the experiment seems to be effective, as the experimental peak output voltage is generally higher than the analytical expectation at the point $\mathrm{Y}=0 \mathrm{~cm}$ (at the centre). The experimental results were influenced by the perpendicular position of the transmitter and the receiver. The large difference in the peak output voltages could also have been caused by external factors, such as the reflection of light from the table surface to the receiver, which would cause the intensity to be higher than expected. The experimental peak voltages shown in Fig. 10 reveal that the output voltage, which is proportional to the light intensity decreased exponentially with the changes of the distance.

\section{ACKNOWLEDGEMENT}

This research project had been carried out in the computer and network security research group laboratory under the Department of Electrical, Electronics and System, Universiti Kebangsaan Malaysia (UKM). This project was sponsored by the Ministry of Higher Education and University Science of Malaysia started on 1st July of 2010. All authors and co-authors are contributed in this project.

\section{REFERENCES}

Conti, J.P., 2008. What you see is what you send. Eng. Techn., 3: 66-69.

Hecht, E., 2008. Optics. 4th Edn., Pearson Education India, San Francisco, London, ISBN: 8131718077 , pp: 650 .

Keiser, G., 2008. Optical Fiber Communications. 1st Edn., Tata McGraw-Hill Education, ISBN: 0070648107, pp: 580.

Khan, J.M. and J.R Barry, 1997. Wireless infrared communications. Proc. IEEE, 85: 265-298.

Komine, T. and M. Nakagawa, 2004. Fundamental analysis for visible-light communication system using LED lights. IEEE Trans. Consumer Elect., 50: 100-107. DOI: 10.1109/TCE.2004.1277847
Langer, K.D., J. Grubor, O. Bouchet, M. El Tabach and J.W. Walewski et al., 2008. Optical wireless communications for broadband access in home area networks. Proceedings of the 10th Anniversary International Conference on Transparent Optical Networks, Jun. 22-26, IEEE Xplore Press, Athens, pp: 149-154. DOI: 10.1109/ICTON.2008.4598756

Langer, K.D., J. Vucic, C. Kottke, L.F. Rosal and S. Nerreter et al., 2009. Advances and prospects in high-speed information broadcast using phosphorescent white-light LEDs. Proceedings of the 11th International Conference on Transparent Optical Networks, Jun. 28-Jul. 2, IEEE Xplore Press, Azores, pp: 1-6. DOI: 10.1109/ICTON.2009.5184994

Nakagawa, M., 2007. Visible light communications. Proceedings of the Conference on Lasers and Electro-Optics (CLEO), May 6-6, Baltimore, Maryland.

Rancourt, J.D., J.B. Hollenhead and L.T. Taylor, 1993. Chemistry of the interface between aluminum and polyester films. J. Adhesion, 40: 267-285. DOI: 10.1080/00218469308031289

Rui, W., D. Jing-yuan, S. An-cun, W. Yong-jie and L. Yu-liang, 2009. Indoor optical wireless communication system utilizing white LED lights. Proceedings of the 15th Asia-Pacific Conference on Communications, Oct. 8-10, IEEE Xplore Press, Shanghai, pp: 617-621. DOI: 10.1109/APCC.2009.5375555

Syuhaimi, M. and K.J. Ab-Rahman, 2011. Experimental works on short range communication systems using photovoltaic based receiver. J. Eng. Applied Sci., 6: 268-276.

Tanaka, Y., S. Haruyama and M. Nakagawa, 2000. Wireless optical transmissions with white colored LED for wireless home links. Proceedings of the 11th International Symposium on Personal Indoor and Mobile Radio Communications, Sep. 18-21, IEEE Xplore Press, London, UK., 1325-1329. DOI: 10.1109/PIMRC.2000.881634

Tanaka, Y., S. Haruyama and M. Nakagawa, 2003. Indoor visible light transmission system utilizing white LED lights. IEICE Trans. Commun., E86: 2440-2454. 